\title{
ADVERSE DRUG REACTIONS IN NEPHROLOGY WARD INPATIENTS OF A TERTIARY CARE HOSPITAL
}

\author{
LISHA JOSHUA, PADMINI D. DEVI, SHOBA GUIDO
}

\section{ABSTRACT}

BACKGROUND: Adverse drug reactions (ADRs) are important causes of hospital admissions and inpatient complications. Renal dysfunction has a role in occurrence of ADRs. AIMS: (1) To study the characteristics of ADRs among inpatients in Nephrology ward of a tertiary care hospital and (2) to compare these characteristics between patients with renal dysfunction and patients with normal renal function in same population of patients with ADRs. MATERIALS AND METHODS: A retrospective study of inpatients with ADRs (J uly 2005-J une 2006) in N ephrology ward of a tertiary care hospital. STATISTICAL ANALYSIS: ADR characteristics were analyzed using descriptive statistics. Comparisons were made between normal renal function group and renal dysfunction group by t-test and Chi-square test. RESULTS: Of 1,464 case records, 244 (17\%) patients were included. Two hundred sixty-seven drugs contributed to 294 ADRs. Serious ADRs accounted for $12 \%$ of the total ADRs. Renal/ electrolyte system (44\%) was the most common organ system involved. Major clinical spectrum of ADRs included acute renal failure (22\%), hypo/ hyperglycemia (13\%), hyper/ hypokalemia (13\%), bone marrow suppression (5\%) and hepatic injuries (4\%). Prednisolone (12\%) was the most commonly implicated drug. Mean time to revert was $13 \pm 7.2$ days. Three patients died. On comparing patients with normal renal function $(n=80)$ with those suffering from renal dysfunction $(n=164)$, polypharmacy, serious ADRs, multiple ADRs, longer time to recover, longer period of hospitalization were found to be more frequent among the renal dysfunction group ( $\mathrm{P}<$ 0.05), with no difference in mortality between groups. CONCLUSIONS: High incidence of ADRs, especially serious and life-threatening ADRs, was noticed. A wide spectrum of ADRs was observed. Renal dysfunction showed a significant impact on various characteristics of ADRs.

Key words: Adverse drug reactions, polypharmacy, prednisolone, renal dysfunction, serious adverse drug reactions

Pharmacovigilance Centre, Department of

Pharmacology, St. John's Medical College, Bangalore, India

\section{Correspondence:}

Dr. Lisha Joshua, Department of Pharmacology,

St. John's Medical College, Bangalore - 560 034, India.

E-mail: drlishaj@yahoo.com

\footnotetext{
Presentation at the $39^{\text {th }}$ Annual Conference of Indian Pharmacological Society; SMS Medical College, Jaipur, Rajasthan, India, on $21^{\text {st }}$ December 2006
}

An adverse drug reaction (ADR) as defined by World Health Organization (WHO) is a noxious, unintended effect of a drug, occurring at normal doses in humans for prophylaxis, diagnosis or therapy of disease or for the modification of physiological function. ${ }^{[1]}$ ADRs are considered as the fourth to sixth leading cause of death among hospitalized patients. ${ }^{[2]}$ About 2.9- 
$5.6 \%$ of all hospital admissions are caused by adverse related events, and approximately $35 \%$ of hospitalized patients experience an ADR. ${ }^{[3]}$ ADRs are associated with significant mortality, morbidity, permanent disability and are a huge economic burden on patients due to prolonged hospitalization. ${ }^{[4]}$

Kidney is the primary route of elimination for drugs and their metabolites. It is important to determine the role of renal dysfunction in the occurrence of ADRs. ${ }^{[5]}$ Studies in India with regard to ADRs among hospitalized patients with renal dysfunction are limited. The objectives of this study were to (1) analyze the characteristics of ADRs among inpatients in the Nephrology ward of a tertiary care hospital and (2) compare these characteristics between patients with renal dysfunction and patients with normal renal function in the same population of patients with ADRs.

\section{MATERIALS AND METHODS}

This retrospective hospital-based study was conducted from $1^{\text {st }}$ July 2005 to $30^{\text {th }}$ June 2006 in the Nephrology ward of a superspecialty, tertiary care, teaching hospital. The case records of all the patients admitted to the Nephrology ward during the study period were obtained from the Medical Records Department and were screened for ADRs based on WHO definitions. ${ }^{[1]}$ Case records of patients who experienced ADRs during their hospital stay, as well as of those who were hospitalized due to ADRs, were identified by the first author and reconfirmed by the second author. Only certain and probable ADRs (based on WHO causality definitions) ${ }^{[1]}$ were included in the study.
The demographic, clinical and treatment data were collected from the inpatient case records using a specially designed pro forma which included age, gender, complete diagnoses, comorbid factors, serum creatinine, blood urea, serum albumin, duration of hospital stay and the outcome. Data collected on adverse drug reactions included drugs received, nature of ADR, drugs implicated, reaction time and time to revert, in accordance to the pro forma.

The patients with the diagnosis of ADRs (only certain and probable) were further subdivided into two groups based on their serum creatinine levels and estimated creatinine clearance (Crockfort and Gault equation) as defined below:

(1) C

Estimated creatinine clearance $(\mathrm{ml} / \mathrm{min})=$ (140- age) (weight in kg)/72 $\times$ serum creatinine. For women, the result was multiplied by $0.85{ }^{[6]}$ Serum creatinine level up to $1.2 \mathrm{mg} / \mathrm{dl}$, creatinine clearance of $120 \mathrm{ml} / \mathrm{min} / 1.73 \mathrm{~m}^{2}$ in men; (100 $\mathrm{ml} / \mathrm{min} / 1.73 \mathrm{~m}^{2}$ in women) were considered as normal. ${ }^{[7]}$

The two groups of patients with ADRs - patients with normal renal function (normal serum creatinine and creatinine clearance) and patients with renal dysfunction (serum creatinine levels over the normal range and creatinine clearance less than normal range) - were compared.

\section{Statistics}

The data collected were subjected to descriptive analyses to study the characteristics of ADRs. Results are expressed as percentages, mean + standard deviation for continuous parametric variables; and median and inter-quartile range (25-75\% IQ) for continuous, nonparametric variables. Comparisons between the two groups were performed using Chi-square and t-test as appropriate. A ' $P$ ' value of less than 0.05 was considered significant. All statistical analyses were performed with statistical software (SPSS 12.0 for Windows).

\section{RESULTS}

Of 1,464 patients admitted to the Nephrology ward during the study period, 244 (17\%) were found to have ADRs. Among these 244 patients, 267 drugs were attributed to cause 294 adverse drug reactions, and 43 (18\%) patients developed more than one ADR. The maximum number of ADRs noted was 4 - in 1 patient. According to the causality assessment, 4 ADRs were classified as 'certain' and 290 as 'probable' associations with the drug. It was found that 222 patients $(91 \%)$ were receiving $>5$ drugs at the time of experiencing an ADR. The maximum number of drugs prescribed for a single patient was 18 . Adults (18-65 years; $72 \%$ ) were most affected by ADRs, followed by the elderly (>65 years; $26 \%$ ). The male-tofemale ratio was 1.7 .

The analyses of clinical characteristics revealed that $131(54 \%)$ patients had $>4$ comorbid factors in the diagnosis. The major comorbid conditions noticed in the study were hypertension 112 (46\%), diabetes mellitus type 260 (26\%), anemia $42(17 \%)$, dyslipidemia $28(12 \%)$ osteoarthritis $24(10 \%)$, coronary artery disease $20(8 \%)$ and lower respiratory tract infections $16(7 \%)$. The maximum number of comorbid factors found in a single patient was 14 .

The details of drug classes, respective organ systems involved and the total number of ADRs are presented in Table 1. The distribution of types of ADRs is presented in Table 2. The frequency of top seven drugs associated with ADRs is shown in Figure 1. Overall, the median reaction time (time taken for the reaction to occur after the last exposure to the suspected drug) of ADRs was 50 days (inter-quartile range 45-57), and the mean time taken for recovery was $13.4 \pm 7.1$ days.

\section{(1)}

The single drug found to be implicated in maximum number of ADRs in a single patien was prednisolone (four ADRs). The clinical spectrum of reactions caused by prednisolone included drug-induced hyperglycemia (25), infection (5), hypertension (3), psychosis (3) hypothalamo pituitary axis suppression (2) gastritis (2) and myopathies (3). The reaction time varied from $6 \mathrm{~h}$ to 2 months.

(n)

The ADRs attributed to diclofenac included acute renal failure (22), gastritis (4). The reaction time varied from 1 to 90 days Nimesulide was suspected in 16 ADRs, and all were acute renal failure cases. The reaction time varied from 2 to 10 days. Enalapril was suspected in 16 reactions, which included hyperkalemia (14), hypotension (1) and dry cough (1), with a reaction time between 5 days and 2 months.

Of the total of 1,464 admissions to the Nephrology ward, serious ADRs were observed in $171(12 \%)$ patients, which accounted for $58 \%$ of the total number of ADRs. Among these, 168 ADRs required hospitalization and 3 resulted in death. Of the 28 life-threatening ADRs, acute renal failure was the most common ADR (11) followed by hyperkalemia (7), pancytopenia (7) and drug-induced hepatic injuries (3). 


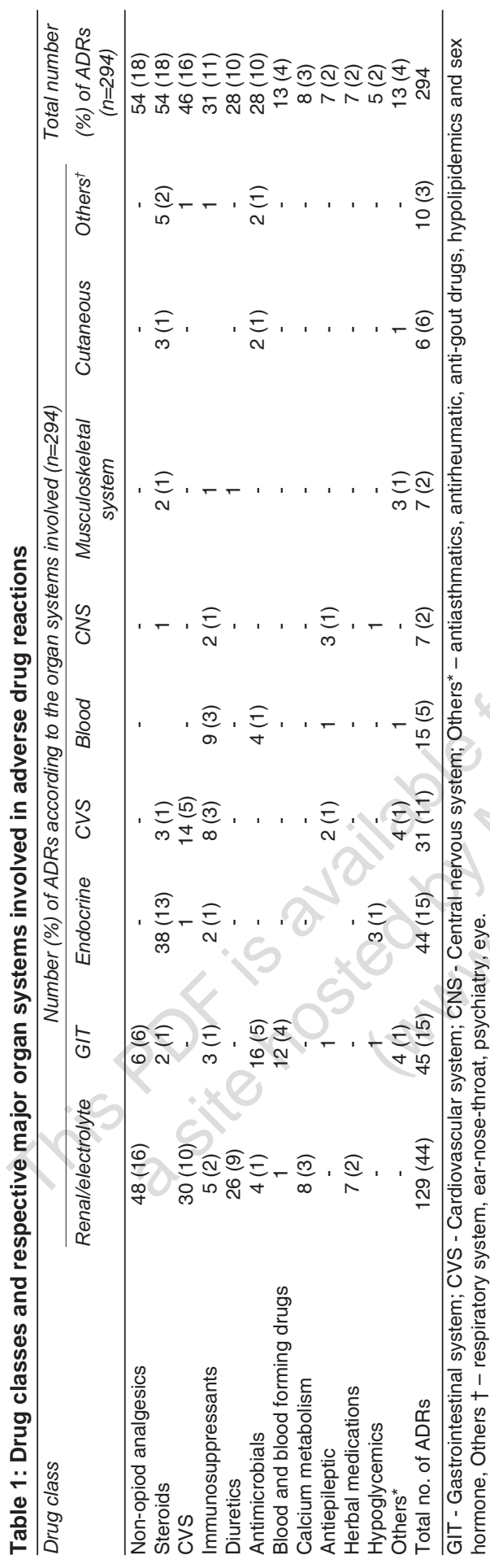

Table 2: Distribution of major types of adverse drug \begin{tabular}{lcc}
\multicolumn{1}{c}{ reactions } & & \\
\hline Type of adverse drug reaction & $\begin{array}{c}\text { Number } \\
(n=294)\end{array}$ & $\%$ \\
\hline Acute renal failure & 64 & 22 \\
Hyperglycemia/hypoglycemia & 39 & 13 \\
Hypokalemia/ hyperkalemia & 38 & 13 \\
Gastritis & 18 & 6 \\
Blood pressure changes & 16 & 5 \\
Pancytopenia/leucopenia/ & & \\
thrombocytopenia & 15 & 5 \\
Hepatic injury/ elevated liver function test & 11 & 4 \\
Hypercalcemia/hypocalcemia & 8 & 3 \\
\hline
\end{tabular}

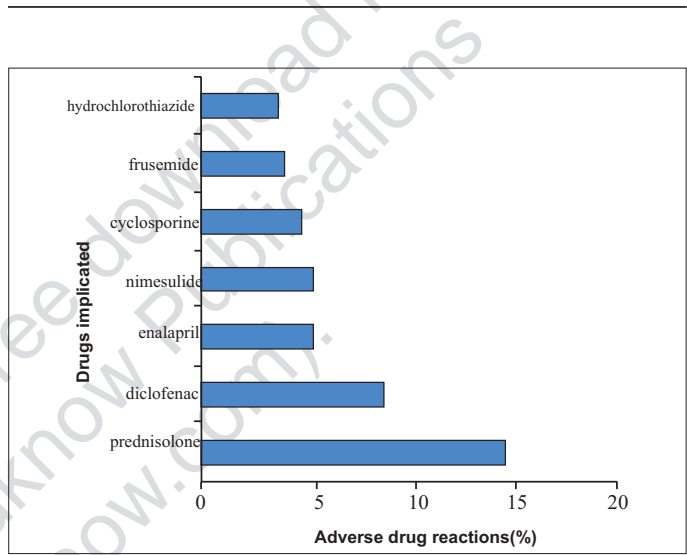

Figure 1: Frequency of top seven drugs associated with ADRs

Out of the total 267 drugs implicated, 7 drugs belonged to herbal medications. Among the remaining 260 drugs, 228 (88\%) were eliminated through the kidney. The most commonly implicated hydrosoluble drugs were steroids $42(18 \%)$, diuretics $22(10 \%)$ and angiotensin converting enzyme inhibitors 15 (7\%).

The mean hospital stay was $8.97 \pm 4.9$ days. Among the 244 patients, $3(1.2 \%)$ patients died with ADRs as one of the causes of death, which accounted for $0.2 \%$ of the total admissions in the Nephrology ward. Among these, multiple ADRs were observed in 2 patients. The ADRs and the drugs suspected in these patients were prednisolone-induced hyperglycemia with hypothalamo-pituitary axis suppression; pancytopenia with antibiotic-induced diarrhea (induced by methotrexate and ciprofloxacin respectively); and fatal bleeding with acute renal failure and hyperglycemia induced by warfarin and prednisolone respectively.

Patients who experienced ADRs in the study (244) were further grouped into two groups based on the renal function: patients with normal renal function $(n=80)$ and patients with renal dysfunction $(n=164)$. The most common clinical diagnoses among patients with normal renal function included adult onse nephrotic syndrome 12 (15\%), membranous glomerulopathy $12(15 \%)$, recurrent urinary tract infection $8(10 \%)$. The comparison of the main characteristics between the two groups of the patients with ADRs is shown in Table 3.

$$
8
$$

Among the patients with renal dysfunction, non-opioid analgesics (49/164; $30 \%$ ) were

$$
20 ; 30 \%)
$$

the main drug class responsible for ADRs. Cardiovascular drugs were the second most common in this group, which resulted in 33 reactions $(20 \%)$. However, steroids were the most common offenders in patients with normal renal function, causing 24 (30\%) reactions. Diuretics and immunosuppressant were responsible for 13 reactions each (16\%) among patients with normal renal function. No significant differences were noted in mortality between the two groups ( $P>0.05$ ). A promp de-challenge of the offending drug was done in all the cases, and the patients were treated appropriately. Serious cases were effectively monitored and managed till discharge.

\section{DISCUSSION}

\section{OPSTION}

The occurrence of ADRs among hospitalized patients in this study was higher $(17 \%)$ compared to those demonstrated in the published literature. ${ }^{[4]} \mathrm{A}$ majority of patients (

\begin{tabular}{|c|c|c|c|}
\hline Characteristic $<$ & $\begin{array}{l}\text { Group with normal } \\
\text { renal function }(n=80)\end{array}$ & $\begin{array}{l}\text { Group with abnormal } \\
\text { renal function }(n=164)\end{array}$ & Comparison \\
\hline Serum creatinine $($ Mean $\pm S D)$ & $1.1 \pm 0.2 \mathrm{mg} / \mathrm{dl}$ & $5.8 \pm 2.9 \mathrm{mg} / \mathrm{dl}$ & $\begin{array}{c}P<0.001 \\
\mathrm{t}=14.246, \mathrm{Cl} 4.6(4.01-5.3)\end{array}$ \\
\hline Serum albumin $<3.2 \mathrm{~g} / \mathrm{dL}$ & $33(41)$ & $59(36)$ & $\begin{array}{c}P=0.5 \\
\chi^{2}=0.43 \mathrm{df}=1 \\
\mathrm{Cl} 0.05(-0.07-0.17)\end{array}$ \\
\hline$>4$ Diagnoses & $30(38)$ & $101(61)$ & $\begin{array}{c}P<0.001 \\
\chi^{2}=9.68 \mathrm{df}=1 \\
\mathrm{Cl} 0.23(0.09-0.37)\end{array}$ \\
\hline$>5$ Prescribed drugs & $68(85)$ & $154(94)$ & $\begin{array}{c}P=0.023 \\
\chi^{2}=3.4 \mathrm{df}=1 \\
\operatorname{Cl} 0.24(0.01-0.46)\end{array}$ \\
\hline > One ADR per patient (multiple ADRs) & $9(11)$ & $34(21)$ & $\begin{array}{c}P=0.05 \\
\chi^{2}=3.0, \mathrm{df}=1 \\
\operatorname{Cl} 0.18(-0.0-0.37)\end{array}$ \\
\hline Serious ADRs & $48(60)$ & $123(75)$ & $\begin{array}{c}P=0.034 \\
\chi^{2}=4.46 \mathrm{df}=1 \\
\mathrm{Cl} 0.17(0.02-0.34)\end{array}$ \\
\hline Time to revert (Mean + SEM) & $8.8 \pm 0.5$ days & $15.7 \pm 0.9$ days & $\begin{array}{c}P<0.001 \\
\mathrm{t}=5.16, \mathrm{Cl} 6.9(4.2-9.5)\end{array}$ \\
\hline Hospital stay (Mean + SEM) & $7.5 \pm 0.7$ days & $9.7 \pm 0.6$ days & $\begin{array}{c}P=0.02, \mathrm{t}=2.225 \\
\mathrm{Cl} 2.2(0.25-4.14)\end{array}$ \\
\hline
\end{tabular}

Table 3: Comparison of characteristics of ADRs between patients with normal renal function and patients

SD - Standard deviation; SEM - Standard error of mean; Cl - Confidence interval; df - Degree of freedom; Figures in parentheses indicate percentage

Indian J Med Sci, Vol. 61, No. 10, October 2007 
affected by ADRs were from the adult population (19-65 years; $72 \%$ ), compared to the elderly (>65 years; $26 \%$ ). This finding differed from reports of other studies. ${ }^{[8,9]}$ Women have been reported to be at greater risk for ADRs. ${ }^{[10]}$ However, in this study a male preponderance was noticed. A majority of patients who experienced an ADR had renal dysfunction $(67 \%)$, and a higher incidence of renal dysfunction among male patients has already been documented. ${ }^{[11]}$

The two important predisposing factors for the occurrence of ADRs observed in this study were extensive polypharmacy ( $91 \%$ receiving $>5$ drugs) and comorbidities (54\%). These findings were found to be significantly higher among patients with renal dysfunction, similar to a previous study. ${ }^{[12]}$ Polypharmacy is linked to many drug-related problems, especially ADRs. ${ }^{[13]}$ Patients with multiple comorbid factors (54\%) were found to be more in this study compared to previous Indian studies (8\%). ${ }^{[4]}$ Concurrent comorbidities such as hypertension (46\%), diabetes (26\%), anemia (17\%) and dyslipidemia $(12 \%)$ could result in polypharmacy and lead to the occurrence of ADRs.

In this study, occurrence of serious ADRs (12\%) and multiple ADRs (18\%) seems to be higher compared to that cited in previously published reports. ${ }^{[2,3]} \mathrm{A}$ wide spectrum of life-threatening ADRs, including acute renal failure, hyperkalemia, pancytopenia and hepatic injuries, was also noticed.

The most common organ system associated with ADRs was renal/ electrolyte system (44\%), similar to the study conducted by Gurwitz et al. ${ }^{[14]}$; but differing from a previous north Indian study, ${ }^{[15]}$ where cutaneous reactions were the highest.

Non-opioid analgesics were implicated in a majority of ADRs (18\%). A previous Indian study had documented aminoglycosides (48\%) as the most common offenders in hospitalacquired renal failure. ${ }^{[16]}$ The majority of the implicated drugs in this study were eliminated through kidney (87\%) and were more frequent among patients with renal dysfunction. This suggests the need to increase the awareness with regard to prescription of hydrosoluble drugs among patients with renal dysfunction. Ore

More than four comorbid factors (61\%), polypharmacy (94\%) and length of hospital stay $(9.72 \pm 0.6$ days) were significantly higher in patients with renal dysfunction [Table 3], similar to a previous study. ${ }^{[12]}$ Multiple ADRs (21\%), serious ADRs (75\%) and time taken to recover from ADRs were also higher in patients with renal dysfunction.

Mortality due to ADRs was $0.2 \%$ of the total admissions, similar to a previous study. ${ }^{[4]}$ The three deaths observed in the study were related to prednisolone-induced hyperglycemia - with hypothalamo-pituitary axis suppression in one patient; pancytopenia with antibioticinduced diarrhea (induced by methotrexate and ciprofloxacin respectively) in one patient; and fatal bleeding with acute renal failure and hyperglycemia induced by warfarin and prednisolone respectively in one patient.

Some limitations are inherent in retrospective studies. When more than one drug was implicated and de-challenged simultaneously, it was difficult to calculate the incidence of

individual implicated drugs. Although we were unable to collect the relevant data of 1,464 patients admitted to the Nephrology ward, we have compared the characteristics of ADRs between patients with normal renal function and those with renal dysfunction in the study population who experienced ADRs. Estimation of GFR or direct measurement of GFR was not performed. However, Cockcroft-Gault formula was used to assess the renal function uniformly for the entire study population.

In conclusion, occurrence of ADRs was found to be higher, especially that of serious ADRs, compared to that reported in previous studies. Older hospitalized patients with renal dysfunction were exposed to increased ADRs, especially to hydrosoluble drugs. Renal dysfunction plays a significant role in occurrence of serious and multiple ADRs. Polypharmacy, comorbidity and longer hospital stay were more frequent in patients with renal dysfunction. Developing newer strategies to report and monitor ADRs, especially to hydrosoluble drugs, in patients with renal dysfunction is highly essential to ensure safe pharmacotherapy.

\section{ACKNOWLEDGMENTS}

\section{COEM}

We acknowledge the help of the staff of the Medical Records Department in carrying out the study.

\section{REFERENCES}

1. Edwards IR, Aronson JK. Adverse drug reactions: Definitions, diagnosis and management. Lancet 2000;356:1255-9.

2. Lazarou J, Pomeranz BH, Corey PN. Incidence of adverse drug reactions in hospitalized patients: A meta-analysis of prospective studies. JAMA
1998;279:1200-5.

3. Murphy BM, Frigo LC. Development, implementation and results of a successfu multidisciplinary adverse drug reaction reporting program in a university teaching hospital. Hosp Pharm 1993;28:1199-204,1240.

4. Ramesh M, Pandit J, Parthasarathi G. Adverse drug reactions in a south Indian hospital their severity and cost involved. Pharmacoepidemio Drug Saf 2003;12:687-92.

5. Jick $H$. Adverse drug effects in relation to renal function. Am J Med 1977;62:514-7.

6. Cockcroft DW, Gault MH. Prediction of creatinine clearance from serum creatinine. Nephron 1976;16:31-41.

7. Watnick S, Morrison G. Kidney. In: Tierney LM, McPhee S, Papadakis MA, editors. Curren medical diagnosis and treatment. Lange McGraw Hill: USA; 2006.

8. Egger T, Dormann H, Ahne G, Runge U, Neubert A, Criegee-Rieck M, et al. Identification of adverse drug reactions in geriatric inpatients using computerised drug database. Drugs Aging 2003;20:769-76.

9. Carbonin P, Pahor M, Bernabei R, Sgadari A. Is age an independent risk factor of adverse drug reactions in hospitalized medical patients? J Am Geriatr Soc 1991;39:1093-9.

10. Tran C, Knowles SR, Liu BA, Shear NH. Gender differences in adverse drug reactions. J Clin Pharmacol 1998;38:1003-9.

11. Jungers $P$, Chauveau $P$, Descamps-Latscha $B$, Labrunie M, Giraud E, Man NK, et al. Age and gender-related incidence of chronic renal failure in a French urban area: A prospective epidemiologic study. Nephrol Dial Transplant 1996;11:1542-6.

12. Corsonello A, Pedone C, Corica F, Mussi C, Carbonin P, Antonelli Incalzi R, et al. Concealed renal insufficiency and adverse drug reactions in elderly hospitalized patients. Arch Intern Med 2005; 165:790-5.

13. Viktil KK, Blix HS, Moger TA, Reikvam A. Polypharmacy as commonly defined is an 
indicator of limited value in the assessment of drug-related problems. $\mathrm{Br} \mathrm{J}$ Clin Pharmacol 2007;63:187-95.

14. Gurwitz JH, Field TS, Harrold LR, Rothschild $\mathrm{J}$, Debellis K, Seger AC, et al. Incidence and preventability of adverse drug events among older persons in the ambulatory setting. JAMA 2003;289:1107-16.

15. Uppal R, Jhaj R, Malhotra S. Adverse drug reactions among inpatients in a north Indian referral hospital. Natl Med J India 2000;13:16-8.

16. Biradar V, Urmila A, Renuka S, Pais P. Clinical spectrum of hospital acquired renal failure: $A$ study from tertiary care hospital. Indian J Nephrol 2004;14:93-6.

Source of Support: Nil, Conflict of Interest: None declared. 